\title{
Meta-Analysis of Clinical Outcomes of Lumbar Fusion Surgical Interventions for Degenerative Spondylolisthesis
}

\author{
Khaled Aneiba1,2*, Sabri Garoushi², Mohammed Elmajee ${ }^{3}$, Mohamed Elsllabi4, Osama A. Tashani² \\ ${ }^{1}$ University Hospital of North Tees, Stockton-on-Tees, UK \\ ${ }^{2}$ Centre for Pain Research, Leeds Beckett University, Leeds, UK \\ ${ }^{3}$ The Royal Oldham Hospital, Oldham, UK \\ ${ }^{4}$ Barnsley District Hospital, Barnsley, UK \\ Email: *Khaled.Aneiba@NTH.NHS.UK
}

How to cite this paper: Aneiba, K., Garoushi, S., Elmajee, M., Elsllabi, M. and Tashani, O.A. (2018) Meta-Analysis of Clinical Outcomes of Lumbar Fusion Surgical Interventions for Degenerative Spondylolisthesis. International Journal of Clinical Medicine, 9, 590-599.

https://doi.org/10.4236/ijcm.2018.97050

Received: March 15, 2018

Accepted: July 21, 2018

Published: July 24, 2018

Copyright $\odot 2018$ by authors and Scientific Research Publishing Inc. This work is licensed under the Creative Commons Attribution International License (CC BY 4.0).

http://creativecommons.org/licenses/by/4.0/

\begin{abstract}
Introduction: Surgical interventions for degenerative spondylolisthesis are varied with comparable claims of success. Fusion based technique is one of the most commonly used surgical interventions in treating this condition. The aim of this meta-analysis is to compare the effectiveness of the Lumbar Interbody Fusion techniques (specifically Posterolateral Interbody approach-PLIF) versus Posterolateral Instrumented Fusion (PLF). The clinical outcomes investigated were: back pain, leg pain, function, Oswestery Disability Index (ODI), Disability Rating Index (DRI), fusion and revision rates if reported. Methods: Combinations of keywords and $\mathrm{MeSH}$ terms, where appropriate, were used to search for studies in Medline via Ovid, Embase, Cochrane Library, and Google scholar. The initial search was conducted on 10 August 2016 and updated on 13 June 2017. Eligibility criteria for the studies to be selected for this meta-analysis were: Randomised Controlled Trials (RCTs), cohort and consecutive cases studies that compared at PLIF versus PLF surgical interventions at the lumbar region. Heterogeneity indicators and Forest plot were computed using RevMan 5. Results: Out of the initial hits of 3021, 5 articles were selected as relevant and assessed for risk of bias and then data was extracted and tabulated. These 5 studies reported data from (900 patients' records, follow up ranges from 6 months to 5 years) undergone one of 2 interventions (PLIF or PLF). The overall effect for ODI and leg pain showed no advantage of any intervention over the other while there was a greater odd ratio of fusion if the operation applied PLIF techniques (Overall $\mathrm{Z}=2.86, p=$ 0.004). Conclusions: There is a need for more high quality clinical trials to compare these two interventions. However, available data indicate that there
\end{abstract}


are comparable results in the main clinical outcomes between PLIF and PLF. PLIF has superior fusion rate which does not seem to affect post-operative pain ratings.

\section{Keywords}

PLIF, PLF, Lumbar Spine, Pain, Spondylolisthesis, Fusion Techniques, Review

\section{Introduction}

Spinal disorders at the lumbar region affect around $11 \%-15 \%$ of the population worldwide [1]. Causes of disc problems at the lumbar region include a degenerative process because of ageing, spinal deformities and instability such as spondylolisthesis. Spondylolisthesis is usually defined as a forward displacement of one of the lower lumbar vertebrae over the vertebra beneath it. When this occurred the resulting pressure on the spinal nerves could lead to pain and other symptoms including a loss of mobility and loss of sensory and motor functions along the nerve pathways [1]. Surgical interventions for degenerative spondylolisthesis are varied with comparable claims of success. Fusion based technique is one of the most commonly used surgical interventions in treating this condition. There are two major approaches for the fusion techniques namely Interbody fusion (specifically Posterolateral Interbody approach-PLIF) and Posterolateral Instrumented Fusion (PLF). In interbody fusions there are three common techniques; Posterior lumbar, Transforaminalor Anterior. However, clinical outcomes of these interventions are inconsistently reported in trials that compare the effectiveness of these interventions. The aim of this meta-analysis is to compare the effectiveness of the Lumbar Interbody Fusion techniques versus Posterolateral Instrumented Fusion (PLF). The clinical outcomes investigated are: back pain, leg pain, function, Oswestery Disability Index (ODI), Disability Rating Index (DRI), fusion and revision rates if reported.

\section{Methods}

Combinations of keywords and MeSH terms, where appropriate, were used to search for studies in Medline via Ovid, Embase, Cochrane Library, and Google scholar. The lists of keywords were (lumbar disc/disk or disc/disk disease, discectomy, posterior, posterolateral, transforaminal, anterior, spondylosis, spondylolisthesis) and (vertebr*, lumbar spine, spine) and (pain, disability, quality of life, outcome). The initial search (No time or language limits) was conducted on 10 August 2016 and updated on 13 June 2017. Eligibility criteria for the studies to be selected for this meta-analysis were: Randomised Controlled Trials (RCTs), cohort and consecutive cases studies that compared at PLIF versus PLF surgical interventions at the lumbar region. Initial screening of titles was conducted by one reviewer (SG) and screening of full articles and data extraction 
was conducted by two reviewers independently with a third reviewer acting as an arbiter in case a disagreement between the two systematic reviewers on the inclusion of a study arises. The decision process to select the relevant studies was as follows:

1) Title and abstracts from databases were managed by Endnote library.

2) If the study is not an RCT, case-control or cohort study involving human patients then it is excluded.

3) If the study does not include a surgical intervention, then it is excluded.

4) If the surgical technique is not a fusion technique, then it is excluded.

Data were extracted by two systematic reviewers and checked by other contributors. The data which extracted from the selected studies included; the study design, grades of the disc disease and the spinal levels affected, number of patients and their age, length of the follow up period after surgery. Data on the fusion rate at the spinal level, outcome score (SF12, SF36), ODI, revision rate, patients' satisfaction and both radiating and back pain on a visual analogue score are also extracted and tabulated.

The risk of bias in the selected articles was assessed using the Cochrane collaborations guidelines. Two reviewers assessed the articles independently and then convened to produce the risk of bias judgment using RevMan 5 software [2]. Any disagreements between the two reviewers were resolved by asking a third reviewer to provide further judgment on the article in question. Heterogeneity indicators were calculated using RevMan 5 and it has been decided if pooling of data on any clinical outcome was required that a fixed effect model should be applied if the heterogeneity indicator $\mathrm{I}^{2}$ was less than $70 \%$ and at least three studies provided suitable data for meta-analysis [3] [4]. Publication bias was assessed visually after producing Funnel plots.

\section{Results}

Out of the initial hits of 3021 hits 46 articles were deemed relevant (Figure 1). Forty-one full length articles were excluded with reasons such as; being a discussion article or report on experiments on cadavers or examination of learning curve of surgeons. Five articles were assessed for risk of bias (Table 1) and then data was extracted and tabulated. These 5 studies reported data from (900 patients' records, follow up ranges from 6 months to 5 years) undergone one of the 2 interventions (see Table 2 for full characteristics of the studies). Patients were matched for sex and age. All studies lacked description of randomisation process and quality of articles ranged from intermediate to poor. Five studies have not reported pain outcomes or Oswestry Disability Index (ODI) scores. Those reported ODI or pain outcomes found no significant differences between the compared interventions. There were no significant differences.

\subsection{Studies' Conclusion}

Authors of 3 out of the five studies included agreed that overall PLIF resulted in 


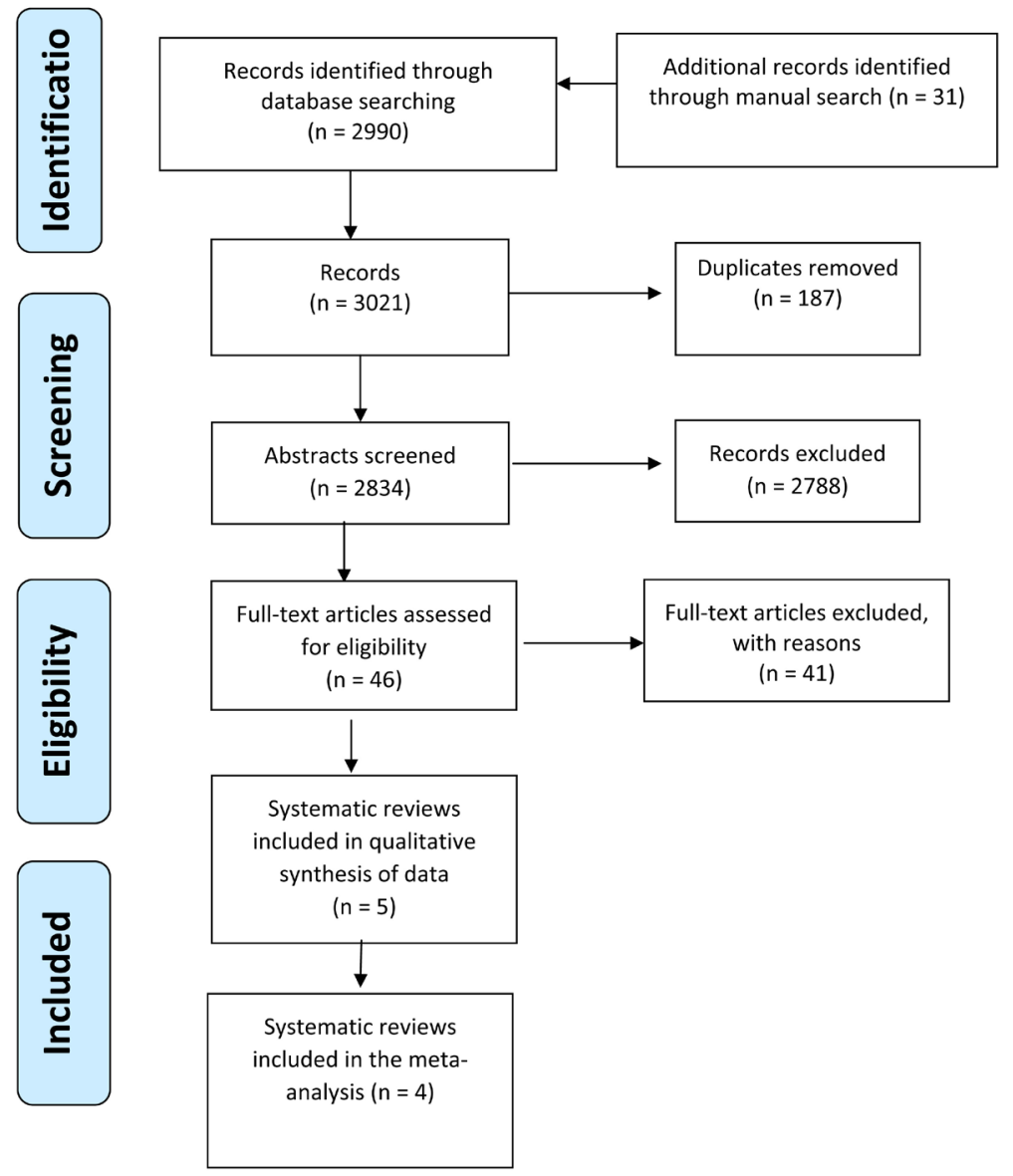

Figure 1. PRISMA flow chart showing results of the selection process.

Table 1. Risk of bias summary: review authors' judgements about each risk of bias item for included studies. Red circle $=$ high risk of bias, Green circle $=$ low risk of bias, Yellow circle $=$ unclear risk of bias.

Sehoux et al.

1-Random sequence generation (selection bias). 2-Allocation concealment (selection bias). 3-Blinding of participants and personnel (performance bias). 4-Blinding of outcome assessment (detection bias). 5-Incomplete outcome data (attrition bias). 6-Selective reporting (reporting bias). 7-Other bias.

similar clinical results as PLF [5] [6] [7] but better fusion rate and better maintenance of reduction is more likely to be obtained post PLIF [5] despite the fact that PLIF could lead to more complications [6]. Farrokhi et al. 2012 
Table 2. Summary of characteristics of studies included and the reported clinical outcomes.

\begin{tabular}{|c|c|c|c|c|c|c|c|c|c|c|c|c|c|}
\hline 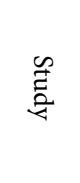 & 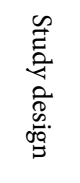 & 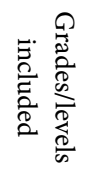 & 兽第 & 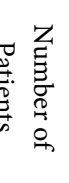 & 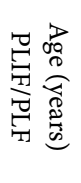 & 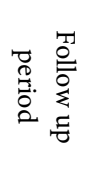 & 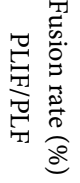 & 易 & & 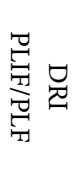 & 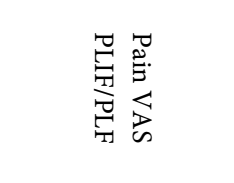 & 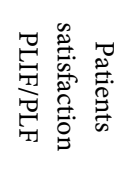 & 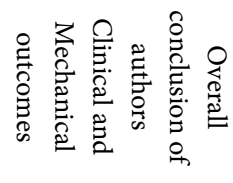 \\
\hline 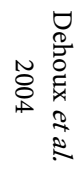 & 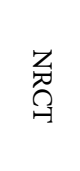 & $\frac{T}{i}$ & $\stackrel{1}{S}$ & Sָ & 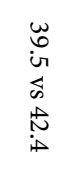 & 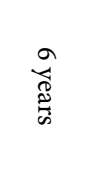 & 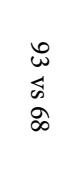 & $\frac{Z}{\Delta}$ & & $\frac{Z}{p}$ & $\frac{Z}{\Delta}$ & 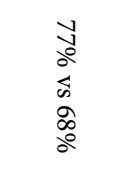 & 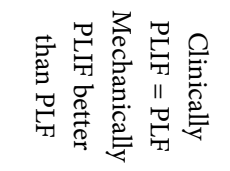 \\
\hline 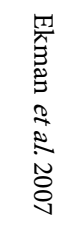 & $\begin{array}{l}Z \\
\stackrel{Z}{\Omega} \\
\stackrel{9}{9}\end{array}$ & 离 & $\begin{array}{l}8 \\
0 \\
0 \\
0\end{array}$ & 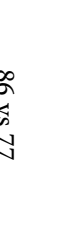 & 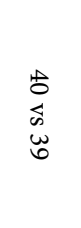 & 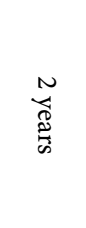 & $\frac{Z}{j}$ & $\begin{array}{l}\tilde{N} \\
\widetilde{\omega} \\
\tilde{N} \\
\tilde{u}\end{array}$ & & 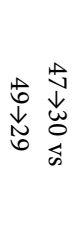 & $\begin{array}{l}w \\
w \\
u \\
s \\
w \\
w\end{array}$ & 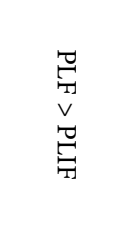 & 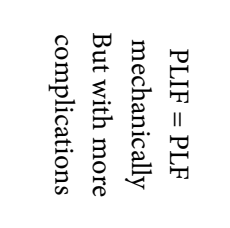 \\
\hline 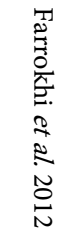 & $\stackrel{\widetilde{\Omega}}{\Omega}$ & $\underbrace{z}_{\substack{w \\
w \\
i}}$ & $\begin{array}{l}\text { 告 } \\
0 \\
0 \\
0\end{array}$ & 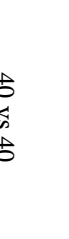 & 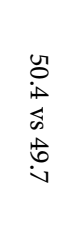 & 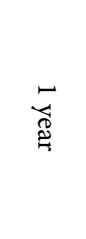 & $\begin{array}{l}\infty \\
\stackrel{\infty}{0} \\
\stackrel{4}{\infty} \\
2 \\
\stackrel{2}{v}\end{array}$ & 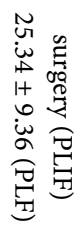 & 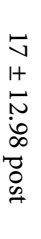 & $\frac{Z}{p}$ & 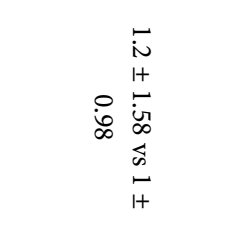 & 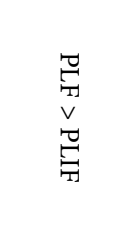 & 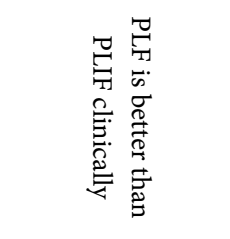 \\
\hline 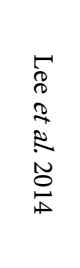 & 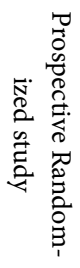 & 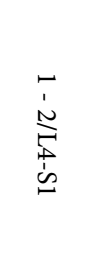 & $\begin{array}{l}\mathbb{A} \\
\Delta \\
\breve{G}\end{array}$ & 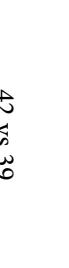 & 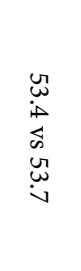 & 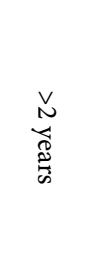 & 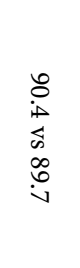 & 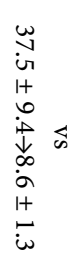 & $\begin{array}{l}\omega \\
\infty \\
0 \\
1 \\
1 \\
0 \\
\dot{1} \\
0 \\
0 \\
1+ \\
1 \\
0\end{array}$ & $\underset{>}{Z}$ & 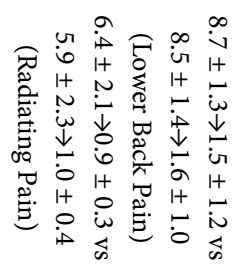 & $\underset{s}{z}$ & 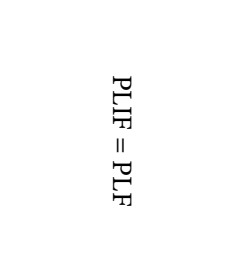 \\
\hline 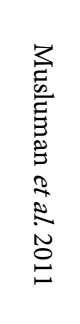 & $\stackrel{\widetilde{\Omega}}{\Omega}$ & 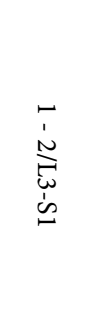 & $\begin{array}{l}\hat{u} \\
\hat{s} \\
\hat{u} \\
\hat{u}\end{array}$ & ک & 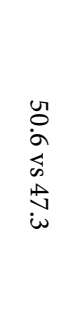 & 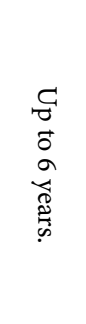 & $\begin{array}{l}2 \\
\swarrow \\
< \\
\infty \\
\infty \\
0\end{array}$ & 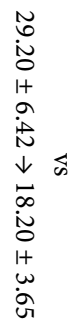 & 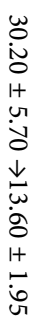 & $\frac{Z}{D}$ & 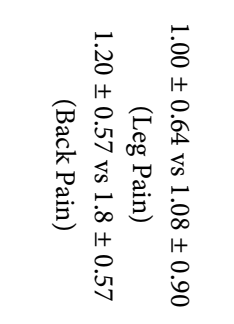 & $\underset{>}{Z}$ & 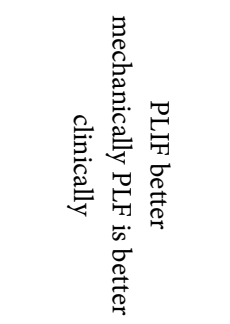 \\
\hline
\end{tabular}

$\mathrm{NRCT}=$ non-randomized controlled trial, $\mathrm{ODI}=$ oswestery disability index, $\mathrm{VAS}=$ visual analogue scale, $\mathrm{DRI}=$ disability rating index.

concluded that PLF provides better clinical outcomes and more improvement in the lower back pain compared to PLIF despite the low fusion rate of PLF [8]. Musluman et al. 2011 suggested that PLIF provided more solid mechanical construct compared to PLF [9]. But PLF exhibited better clinical outcomes at an earlier stage, including improvements in quality of life pain relief and functional ability.

There is also an agreement between all the studies that additional studies with a larger sample size should be performed to better understand the clinical and 
radiological outcomes of both techniques.

\subsection{Meta-Analysis Findings}

It was very difficult to judge if there was publication bias because of the low number of studies in different outcomes. It was possible to pool data in three outcomes Oswestery Disability Index (ODI) (Figure 2), leg pain (Figure 3) and fusion rate (Figure 4). See Appendix 1 for full calculations and data. The overall

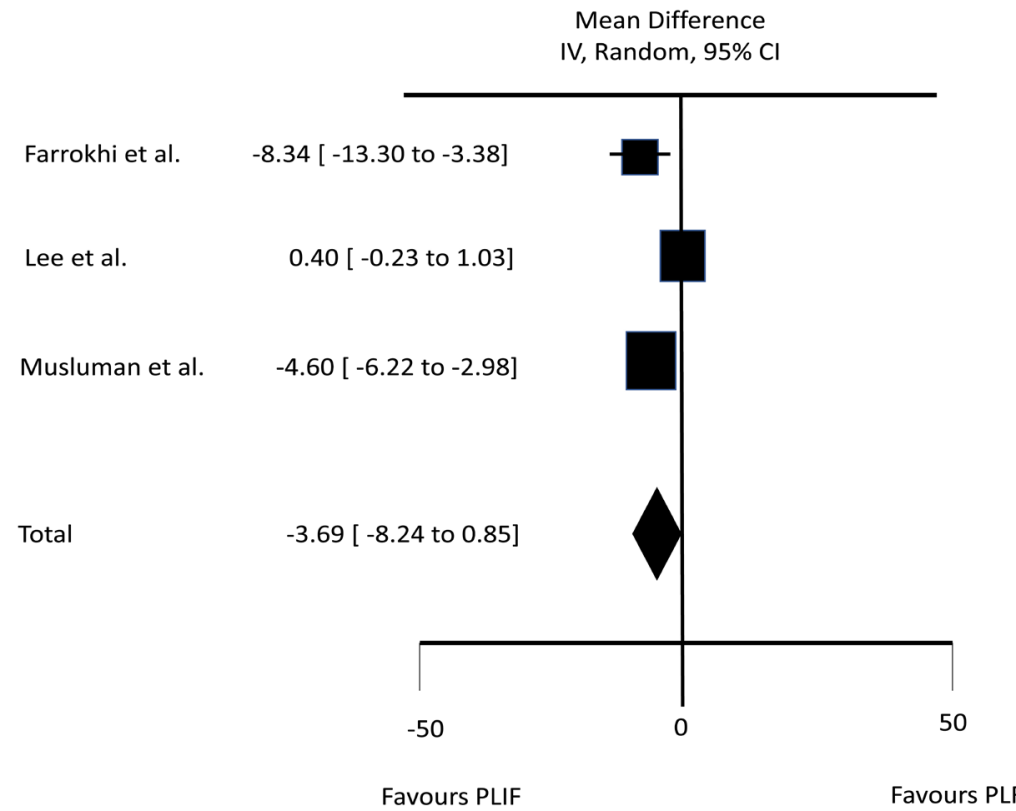

Figure 2. Forest plot of comparison of ODI post-surgery.

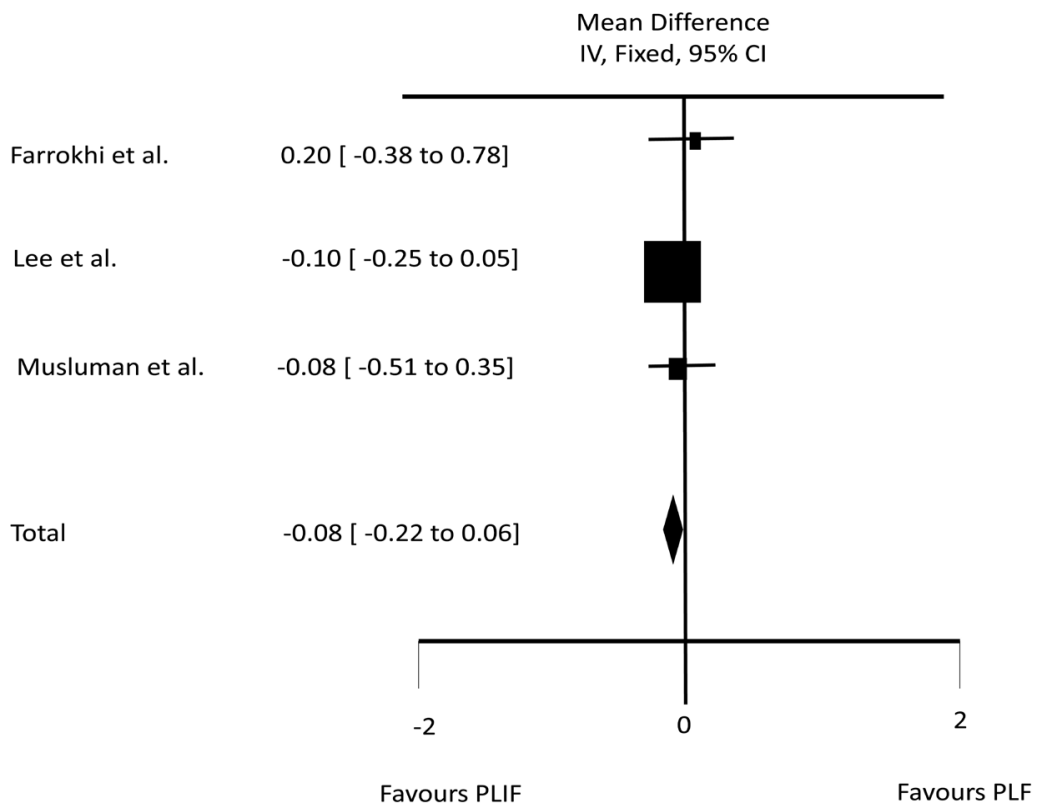

Figure 3. Forest plot of comparison between visual analogue scale (0 to 10) post operations of leg pain. 


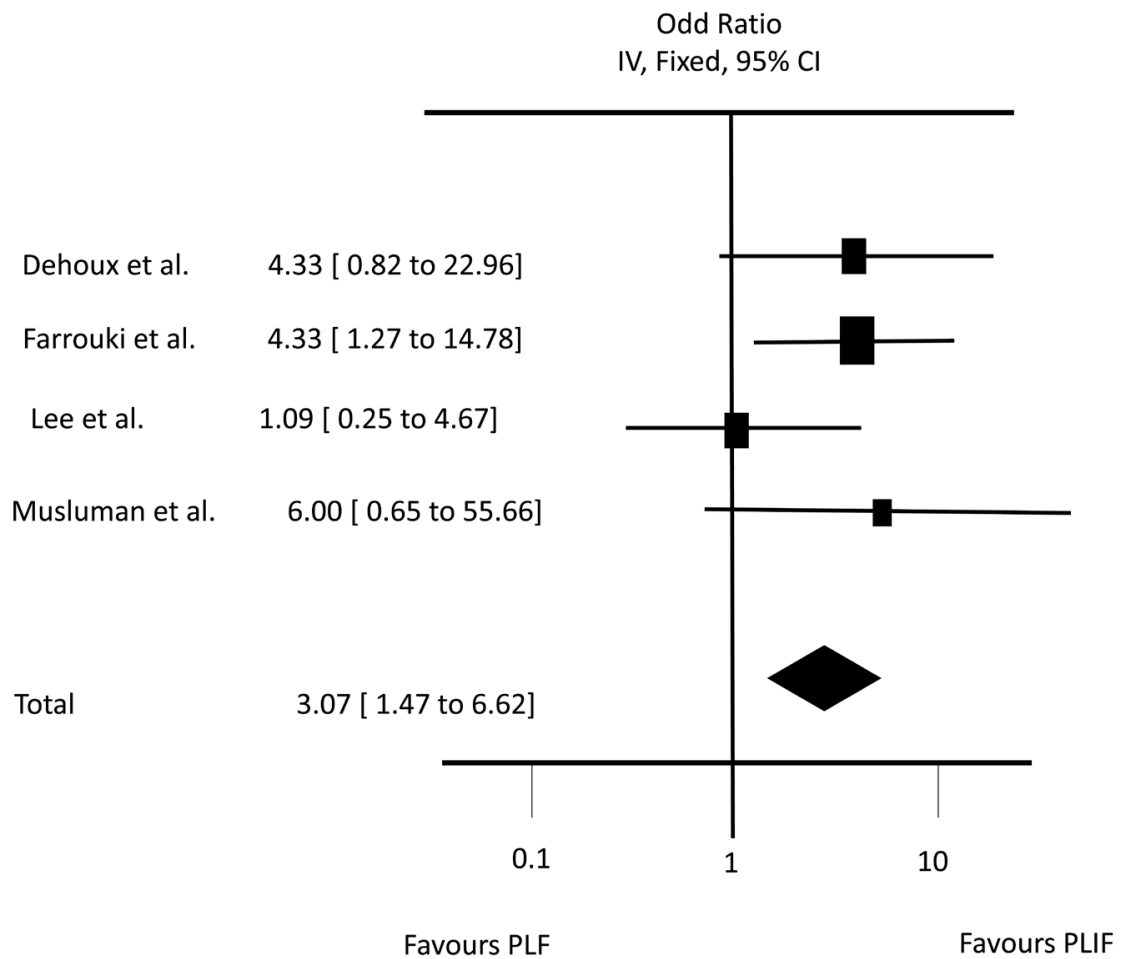

Figure 4. Forest plot of comparison between the odd ratio of fusion rate outcome in the two interventions. There is a greater odd ratio of fusion if the PLIF technique was applied (Overall $\mathrm{Z}=2.86, p=0.004$ ).

effect for ODI and leg pain showed no advantage of any intervention over the other while there was a greater odd ratio of fusion if the operation applied PLIF techniques (Overall $\mathrm{Z}=2.86, p=0.004$ ).

\subsection{Complications}

Overall there were slightly more complications post PLIF operations. There is some evidence that PLIF procedure resulted in more bleeding and is more invasive [6] and the leak of cerebrospinal fluid was $14 \%$ more in post PLIF than post PLF with a slightly greater risk of infection and slightly greater probability of permanent motor impairment in post PLIF patients [8]. Blood Loss (ml) $360 \pm$ 30 vs $350 \pm 25$, Operation time (Hours) $2.6 \pm 0.3$ vs $2.1 \pm 0.2$, Hospital stay (Days) $4.7 \pm 2.2$ vs $4.8 \pm 1.7$, incision length $(\mathrm{cm}) 8.6 \pm 1.7$ vs $8.1 \pm 1.5$.

\section{Discussion}

Findings from the 5 articles selected and analysed in this review suggested that fusion rate is slightly better post PLIF despite the likelihood of higher complications with this intervention. In the four studies that reported fusion rates, three studies reported a fusion rate post PLIF to be at least $16 \%$ more efficient than post PLF. This finding is in agreement with the meta-analysis outcome of Ye et al. 2013, who found that PLF generally produced less fusion rates than PLIF [10]. Clinically the fusion rate post lumbar surgery for spondylolisthesis is the main 
goal of the intervention as it usually linked to the favourite clinical outcomes [11]. However, as far as other clinical outcomes are concerned the two interventions are comparable to each other. While the selected studies did not discussed reasons behind a choice of one intervention over the other it is clear that surgeon preference and experience and anatomical consideration determine what type of surgery is performed.

Despite the significant difference between PLIF and PLF in the fusion rate the pain outcomes, whether it was leg or back pain, was similar after the surgery in the two groups of patients. This was also the findings of [10] and [12]. This is unexpected in the case of back pain as a better fusion rate should have an impact on the persistence of this type of pain. Differences between the follow-up in the selected studies only partially could explain why there was no differences between PLIF and PLF in visual analogue scale of back pain after surgery. However, the similarity of the pain ratings between the two interventions can be explained by the fact that the two surgeries achieve satisfactory spinal nerve root decompression. Our findings should be cautiously interpreted because the studies selected for this meta-analysis lacked description of randomisation process and not all outcomes were reported. While it is accepted that blinding surgeons is impossible the studies failed to blind patients. Other limitation of this meta-analysis is the differences between studies in the follow-up period.

\section{Conclusion}

There is a need for more high quality clinical trials to compare these two interventions. However, available data indicate that there are comparable results in the main clinical outcomes with PLIF providing superior fusion rate which does not seem to affect post-operative pain ratings.

\section{Conflicts of Interest}

The authors declare no conflicts of interest regarding the publication of this paper.

\section{References}

[1] Ha, K.Y., Na, K.H., Shin, J.H. and Kim, K.W. (2008) Comparison of Posterolateral Fusion with and without Additional Posterior Lumbar Interbody Fusion for Degenerative Lumbar Spondylolisthesis. Journal of Spinal Disorders \& Techniques, 21 229-234. https://doi.org/10.1097/BSD.0b013e3180eaa202

[2] Higgins, J.P. and Green, S. (2011) Cochrane Handbook for Systematic Reviews of Interventions. John Wiley \& Sons, Hoboken.

[3] Higgins, J.P.T., Thompson, S.G., Deeks, J.J. and Altman, D.G. (2003) Measuring Inconsistency in Meta-Analyses. BMJ, 327, 557-560. https://doi.org/10.1136/bmj.327.7414.557

[4] Harbord, R.M. and Higgins, J. (2008) Meta-Regression in Stata. The Stata Journal, 8, 493-519.

[5] Dehoux, E., Fourati, E., Madi, K., Reddy, B. and Segal, P. (2005) Posterolateral ver- 
sus Interbody Fusion in Isthmic Spondylolisthesis: Functional Results in 52 Cases with a Minimum Follow-Up of 6 Years. Acta orthopaedica Belgica, 70, 578-582.

[6] Ekman, P., Moller, H., Tullberg, T., Neumann, P. and Hedlund, R. (2007) Posterior Lumbar Interbody Fusion versus Posterolateral Fusion in Adult Isthmic Spondylolisthesis. Spine, 32, 2178-2183. https://doi.org/10.1097/BRS.0b013e31814b1bd8

[7] Lee, G.W., Lee, S.M., Ahn, M.W., Kim, H.J. and Yeom, J.S. (2014) Comparison of Posterolateral Lumbar Fusion and Posterior Lumbar Interbody Fusion for Patients Younger than 60 Years with Isthmic Spondylolisthesis. Spine, 39, E1475-E1480. https://doi.org/10.1097/BRS.0000000000000596

[8] Farrokhi, M.R., Rahmanian, A. and Masoudi, M.S. (2012) Posterolateral versus Posterior Interbody Fusion in Isthmic Spondylolisthesis. Journal of Neurotrauma, 29, 1567-1573. https://doi.org/10.1089/neu.2011.2167

[9] Musluman, A.M., Yilmaz, A., Cansever, T., Cavusoglu, H., Colak, I., Genc, H.A., et al. (2011) Posterior Lumbar Interbody Fusion versus Posterolateral Fusion with Instrumentation in the Treatment of Low-Grade Isthmic Spondylolisthesis: Midterm Clinical Outcomes. Journal of Neurosurgery: Spine, 14, 488-496. https://doi.org/10.3171/2010.11.SPINE10281

[10] Ye, Y.P., Xu, H. and Chen, D. (2013) Comparison between Posterior Lumbar Interbody Fusion and Posterolateral Fusion with Transpedicular Screw Fixation for Isthmic Spondylolithesis: A Meta-Analysis. Archives of Orthopaedic and Trauma Surgery, 133, 1649-1655. https://doi.org/10.1007/s00402-013-1868-5

[11] Kunze, B., Drasseck, T. and Kluba, T. (2011) Posterior and Transforaminal Lumbar Interbody Fusion (PLIF/TLIF) for the Treatment of Localised Segment Degeneration of Lumbar Spine. Zeitschrift für Orthopädie und Unfallchirurgie, 149, 312-316. https://doi.org/10.1055/s-0030-1250689

[12] Zhou, Z.J., Zhao, F.D., Fang, X.Q., Zhao, X. and Fan, S.W. (2011) Meta-Analysis of Instrumented Posterior Interbody Fusion versus Instrumented Posterolateral $\mathrm{Fu}$ sion in the Lumbar Spine. Journal of Neurosurgery. Spine, 15, 295-310. https://doi.org/10.3171/2011.4.SPINE10330 


\section{Appendix 1: Data Used to Produce Effect Size and Forest Plot}

Table (a). Data used to produce Forest plot output in RevMan 5 to compare ODI post-surgery.

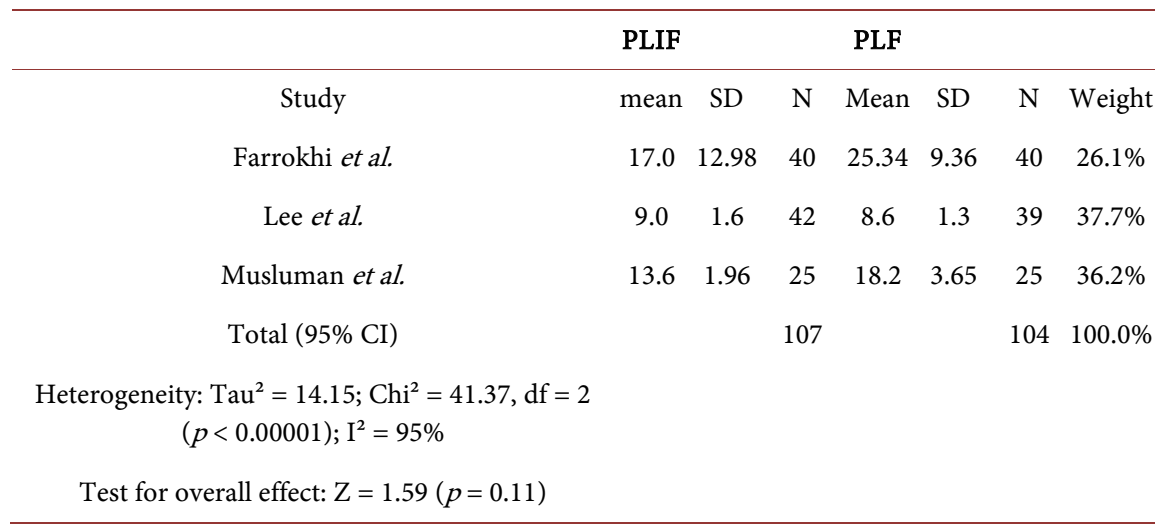

Table (b). Data used to produce Forest plot output in RevMan 5 to compare between visual analogue scale (0 to 10 ) post operations of leg pain.

\begin{tabular}{|c|c|c|c|c|c|c|c|}
\hline & PLIF & & & PLF & & & \\
\hline Study & mean & $\mathrm{SD}$ & $\mathrm{N}$ & Mean & SD & $\mathrm{N}$ & Weight \\
\hline Farrokhi et al. & 1.2 & 1.58 & 40 & 1 & 0.98 & 40 & $6 \%$ \\
\hline Lee et al. & 0.9 & 0.3 & 42 & 1 & 0.4 & 39 & $83.3 \%$ \\
\hline Musluman et al. & 1 & 0.64 & 25 & 1.08 & 0.9 & 25 & $10.7 \%$ \\
\hline Total $(95 \% \mathrm{CI})$ & & & 107 & & & 104 & $100.0 \%$ \\
\hline \multicolumn{8}{|l|}{$\begin{array}{c}\text { Heterogeneity: } \mathrm{Chi}^{2}=0.97, \mathrm{df}=2(p=0.62) \\
\qquad \mathrm{I}^{2}=0 \%\end{array}$} \\
\hline Test for overall effect: $Z=1.11(p=0.27)$ & & & & & & & \\
\hline
\end{tabular}

Table (c). Data used to produce Forest plot output in RevMan 5 to compare log odd ratio of Fusion Rate outcome. There is a greater odd ratio of fusion if the PLIF techniques were applied. (Overall $\mathrm{Z}=2.86, p=0.004$ ).

\begin{tabular}{cccc}
\hline & Log OR & SE & Weight \\
\hline Dehoux et al. & 1.5 & 0.85 & $21.2 \%$ \\
Farrokhi et al. & 1.5 & 0.63 & $39.2 \%$ \\
Lee et al. & 0.08 & 0.74 & $27.7 \%$ \\
Musluman et al. & 1.8 & 1.14 & $11.9 \%$ \\
Heterogeneity: Chi $^{2}=2.76, \mathrm{df}=2(p=0.43) ; \mathrm{I}^{2}=0 \%$ & & & \\
Test for overall effect: $\mathrm{Z}=2.86(p=0.004)$ & & &
\end{tabular}

